\title{
Histological staining approaches for high-quality imaging of Actinia equina and Anemonia sulcata anatomy
}

\author{
J. Gadelha***, F. Morgado* and A.M.V.M Soares *
}

*CESAM \& Departamento de Biologia, Universidade de Aveiro, Portugal

**CAPES Foundation, Ministry of Education of Brazil, Brasília - DF 70040-020, Brazil

Sea anemones species identification of can be difficult, especially in the field. The taxonomic keys currently utilised are based mainly on histological differences and therefore require collection of whole animals. Furthermore, histological analysis of sea anemones requires considerable expertise and worldwide sea anemones histological descriptions are still limited. Here it is reported a detailed histological description of the internal structure of the two most conspicuous species in tidal pool communities in European shores, Actinia equina and Anemonia sulcata. This species description makes them excellent models for studying reproductive strategies that are instrumental in establishing and maintaining new populations. Therefore they are potential indicator species important both for micro and macro scale monitoring programs. The anatomy and histology of main organs of the studied sea anemones were described. The different colour morphs of the species were studied. Fine histological details of their muscular and digestive systems and appendages, down to the cellular level, were studied. They are two-germinal layer animals and possess radial symmetry in body form. Samples of the main organs of sea anemones including epidermis, tentacles, mesentery, gonads and digestive tract (mouth, stomodaeum and gastrovascular cavity) were processed for histological examination according to procedures described elsewhere [1,2]. The epithelia were composed of epithelio-muscular cells, in which many gland cells were distributed. Three types of cnidoblasts (nematooysts) were observed in the epithelioma of the tentacles, mesentery filament and stomodaeum. Cnidoblasts in different places have different functions. The gonads were of the follicular type and were composed of germ cells and nutritional cells. The investigation of the internal organization of invertebrate communities provides important information on the organism's biology with special interest for the study of ecological processes to evaluate their reproductive modes in order to understand how they may reflect their dispersal strategies $[1,2]$. Traits that influence reproduction in animal and plant species have been a central focus of evolutionary biology. Therefore, considering their basal position in evolution, the Anthozoa (Cnidaria) provide important models for further understanding processes affecting reproductive strategies in the eumetazoan (i.e., cnidarian-bilaterian) ancestor and in modern vertebrates.

\section{References}

1. Peters E.C. et al., In: OSTRANDER, G. K. - Techniques in aquatic toxicology. Taylor Francis. ISBN 9781566706643, p. 653-686, 2005.

2. Vanpraet M., Cahiers de Biologie Marine, 19:415, 1978.

This work was supported by the Portuguese Foundation for the Science and Technology - Portugal and FEDER funds, through the Projects: PTDC/MAR/464729/2006 and FCT/CNPq (Brazil), Project 6818, Programme 19/ 004. 

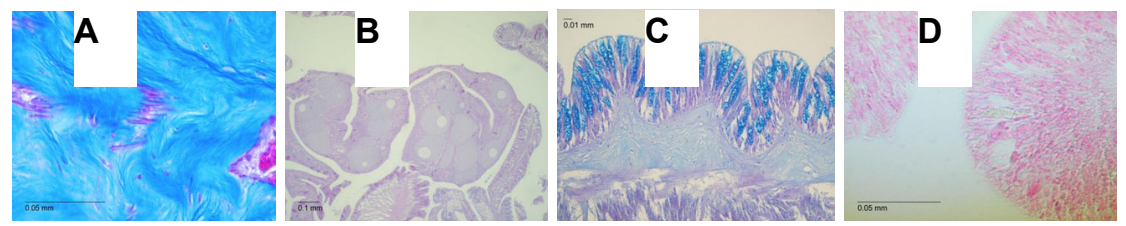

Figure1. Histological staining approaches; A-Details of Mesoglea evidencing fibres (Trichrome Masson) (Actinia equina); B-Mesenterial details (PAS) (Anemonia sulcata); C- Epidermical mucous cells (PAS with Alcian Blue) (Actinia equina); D-Details of mesenterial filaments to localize free ferric ions (Perls) (Actinia equina).
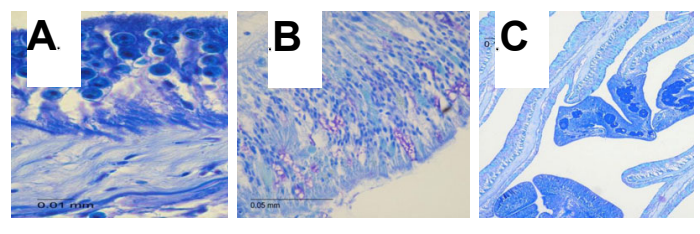

Figure 2- Histological staining approaches; A- Gastrodermal mesenterial with zooxanthellae and amebocites (Toluidine Blue) (Anemonia sulcata). B- Detail of epidermis with secretory cells (Actinia equina). C- Structural details of gonads and muscles.

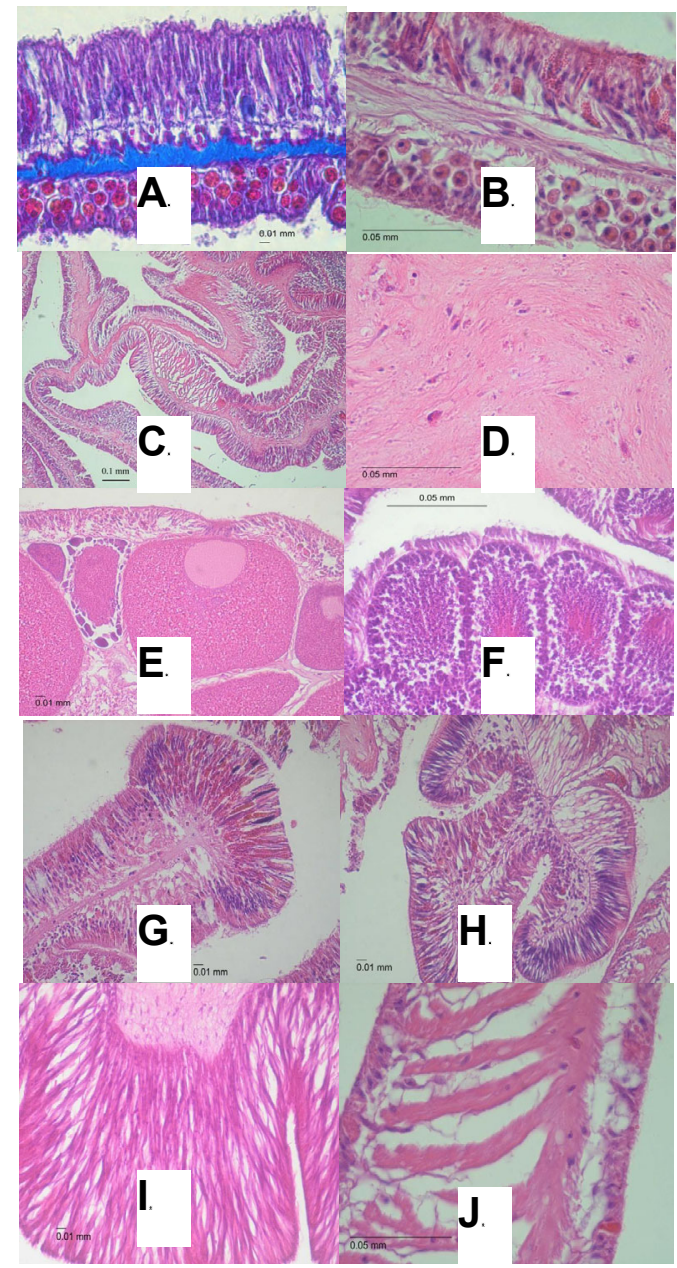

Fig. 3- Microscopical anatomy of Anemonia sulcata. A e B - Tentacle; C - Marginal sphincter; D - Detail of mesoglea; $\mathrm{E}$ - Female gonad vitellogenic oocites with visible trofoneme; $\mathrm{F}$ - Male gonads, in a medial stage of maturation, $\mathrm{G}$ Mesenterial filaments, $\mathrm{H}$ - Trilobate mesenterial filaments; I - Epidermis of basal ring J - Basal muscle. 